\title{
Sistema de Información Estratégica para la Gestión Universitaria en la Universidad de Otavalo (Ecuador)
}

\author{
Luis A. Acosta(1), Francisco A. Becerra ${ }^{(2)}$ y Diego Jaramillo(1) \\ (1) Universidad de Otavalo, Campus: Cdla Imbaya. Av. de los Sarances s/n y Pendoneros, Otavalo, \\ Ecuador. (e-mail: lacosta@uotavalo.edu.ec, djaramillo@otavalo.edu.ec) \\ (2) Universidad de Cienfuegos, Cuatro Caminos, Cienfuegos, Cuba (e-mail: fbecerra@ucf.edu.cu)
}

Recibido Ago. 24, 2016; Aceptado Oct. 27, 2016; Versión final Ene. 10, 2017, Publicado Abr. 2017

\begin{abstract}
Resumen
El objetivo de este estudio fue analizar las características fundamentales de un Sistema de Información Estratégica para la Gestión Universitaria (SIE-GU), diseñado e implementado en la Universidad de Otavalo para apoyar la planificación estratégica, la evaluación institucional y la toma de decisiones. Se aplicó una metodología sustentada en diez etapas, que consideró el análisis de la planificación estratégica, la disponibilidad y la necesidad de información, su compatibilización, la identificación de los principales procesos universitarios, y la propuesta de un SIE-GU, el cual estuvo formado por cuatro módulos principales: academia, investigación vinculación y administración, y se basó en el modelo cliente-servidor. Los resultados de la implementación del SIE-GU contribuyeron al perfeccionamiento de la gestión universitaria, mediante la automatización de la mayoría de los procesos y actividades vinculados con la planificación y la evaluación institucional y agilizaron considerablemente el procesamiento y análisis de la información útil para la toma de decisiones.
\end{abstract}

Palabras clave: gestión universitaria; sistemas de información estratégica; planificación estratégica; evaluación institucional; toma de decisiones

\section{Strategic Information System for University Management at the University of Otavalo (Ecuador)}

\begin{abstract}
The objective of this study was to analyze the fundamental characteristics of a Strategic Information System for University Management (SIE-GU), designed and implemented at the University of Otavalo in Ecuador to support strategic planning, institutional evaluation and decision making. A ten-stage methodology was applied, which considered the analysis of strategic planning, the availability and the need for information, its compatibility, the identification of the main university processes, and the proposal of a SIE-GU, which was formed by four main modules: academia, research linking and administration, and was based on the clientserver model. The results of the implementation of the SIE-GU contributed to the improvement of university management, by automating most of the processes and activities related to planning and institutional evaluation, and considerably facilitated the processing and analysis of information useful for decision making.
\end{abstract}

Keywords: university management; strategic information systems; strategic planning; institutional evaluation; decision making 


\section{INTRODUCCIÓN}

Uno de los retos más desafiantes de la educación superior en la actualidad es el perfeccionamiento de la gestión universitaria y de cada uno de los procesos que la integran. En la Conferencia Mundial sobre la Educación Superior "La educación superior en el siglo XXI: Visión y acción", organizada en París por la UNESCO en octubre de 1998 se expresó el deber de generalizar en la mayor medida posible la utilización de las nuevas tecnologías para que ayuden a los establecimientos de educación superior a reforzar el desarrollo académico, a ampliar el acceso, a lograr una difusión universal y extender el saber, y a facilitar la educación durante toda la vida. Los gobiernos, los establecimientos de enseñanza y el sector privado deberán procurar, enfatiza el informe, en que se faciliten en un nivel suficiente infraestructuras de informática y de redes de comunicaciones, servicios informáticos y formación de recursos humanos (UNESCO, 1998).

Muchas veces se confunde el concepto de tecnología de la información ( $\mathrm{TI}$ ) con el de sistemas de información (SI). En las definiciones conceptuales del término "tecnología de la información", generalmente se hace énfasis en determinados aspectos, como pueden ser: el tratamiento de la información, la solución de problemas, la toma de decisiones y la integración de las tecnologías, entre otros. Al respecto, Mansfield expone una definición bastante amplia cuando afirma que las TI se pueden definir como el conjunto de desarrollos tecnológicos relacionados con la elaboración, transmisión, manipulación y presentación de datos, que sobre la base del microprocesador se aplican en las áreas de la comunicación, el cálculo y el control (Mansfield, 1984).

Para obtener el máximo de las tecnologías de la información es necesario gestionarla eficientemente y ello conduce al análisis del concepto de sistemas de información. Muy precisa es la definición que los considera como un conjunto de personas, datos, procesos y tecnología de la información que interactúan para recoger, procesar, almacenar y proveer la información necesaria para el correcto funcionamiento de la organización (Whitten, et al., 2004). De ahí, la atención que merecen por constituir un soporte imprescindible que permite desarrollar y fortalecer el proceso de enseñanza-aprendizaje; organizar las investigaciones en correspondencia con los intereses de la propia institución y de su entorno; automatizar los procesos administrativos y las actividades de vinculación con la comunidad. Muchas instituciones educativas han encontrado en las TI un potente aliado (Martin-Gutiérrez, et al., 2015), al punto que el progresivo avance de las $\mathrm{TI}$ se posiciona como una de las principales fuentes de innovación y de ventaja competitiva en los sectores que las han implementado, como es el caso de la educación (Dastan, et al., 2011)

La propia evolución de las TI ha condicionado el desarrollo de una tipología de SI en paralelo. Para Senn (1992), desde la perspectiva de la toma de decisiones, los SI pueden ser: de Procesamiento de Datos (TPSTransactional Processing Systems), Sistemas de Información para la Administración o Gerenciales (MISManagement Information Systems), Sistemas de Soporte a la Toma de Decisiones (DSS- Decision Support Systems), Sistemas de información para ejecutivos (EIS- Executive Information Systems) y Sistemas Expertos o sistemas basados en el conocimiento (WKS-Knowledge Working Systems).

Lo que hoy es conocido como "Sistema de Información Gerencial" ha ido evolucionando con el transcurso del tiempo. En una primera instancia, los canales de comunicación eran informales en estructura y utilización; sin embargo, con la aparición y el uso en masas de la informática, se transformaron en "Sistemas de Procesamiento Electrónico de Datos". Posteriormente, dieron lugar al concepto de "Sistema de Información Basado en Computadoras", que se popularizaron como "Sistemas de Información Gerencial". Al introducir en esta terminología el concepto de "Estrategia", entendida como la formulación, ejecución y evaluación de acciones que permitirán que una organización logre sus objetivos, conceptualmente se deriva a los conocidos Sistemas de Información Estratégica (SIE), que forman parte del ser de la organización, bien porque supone una ventaja competitiva por sí mismo, o bien porque está unido de una forma esencial al negocio y aporta un atributo especial a sus procesos y a la toma de decisiones (Puello, et al., 2013).

En la planificación institucional de la Universidad de Otavalo (UO), Ecuador, se establece como una de sus metas más importantes para el período 2015-2020, la elaboración e implementación de un SIE, que contribuya a proporcionar la información necesaria y oportuna para la toma de decisiones. Por ello, se avanza en la formación de los directivos y profesores en temas relacionados con la importancia de la dirección estratégica y la evaluación institucional y se fortalece el trabajo de dirección en equipos, el alineamiento de las estrategias, objetivos estratégicos y la visión de futuro, compartida e integrada a nivel institucional mediante el uso de las TI (Ali, et al., 2014). Se reconoce como una de las principales fortalezas de la institución, la tecnología de punta aplicada a la educación en todas las aulas, la accesibilidad plena a internet e intranet, así como el compromiso y la motivación de la mayoría de los profesores con la visión y proyección estratégica institucional (Martelo, et al., 2015). 
Sin embargo, se detectan un grupo de problemas o barreras (Hosseini et al., 2012) de diversa índole que exigen la búsqueda de soluciones acorde a las propias exigencias actuales de la gestión universitaria y de los retos actuales y futuros de la nueva universidad ecuatoriana. Luego, la cuestión esencial a analizar en este artículo está relacionada con las características debe tener un SIE aplicado a la gestión universitaria, de modo que permita sustentar de manera efectiva la planificación, evaluación y toma de decisiones en la universidad, para contribuir eficazmente al mejoramiento de la calidad de los procesos universitarios.

Los argumentos que justifican la necesidad de disponer de este sistema son: la inexistencia de un mecanismo único para la recolección de la información y la poca eficacia en su tratamiento, análisis y distribución; la insuficiente racionalidad y eficiencia en el uso y manejo de la información en el tiempo adecuado, de manera que permita dar respuesta a los problemas que se presentan con ayuda de la tecnología; la comunicación ineficaz entre la dirección, la secretaría académica y el resto de las áreas de la institución; la incipiente formación en conocimientos en el área del saber de la informática de algunos de los protagonistas del proceso; la utilización de diversos criterios para la clasificación de la información, la duplicidad de bases de datos, el deficiente control de la fiabilidad de la información y la escasa cultura del uso de la misma.

Existen tres elementos claves en la gestión estratégica: la excelencia, la innovación y la anticipación (organización proactiva). Teniendo en cuenta que la gestión de la información incorpora los elementos de la gestión de la organización, también puede hablarse de gestión estratégica de la información, cuando la organización que la maneja le permite proyectarse hacia la consecución de la visión (el futuro), se pueden tomar decisiones anticipadas y se hace proactiva ante cualquier situación del entorno. Sin duda, el avance en la implementación de los SIE y su aplicación a la gestión evoluciona más en el ámbito empresarial, pero también resulta muy pertinente para la gestión universitaria (Martínez, 2006).

En el contexto universitario, las TI permiten apoyar el proceso enseñanza-aprendizaje en un entorno virtual (desarrollo de los programas de las asignaturas de cada carrera), optimizar los procesos que se llevan a cabo en las distintas áreas del conocimiento, tanto en el pregrado como en la continuidad de estudios, potenciar la calidad de las investigaciones en las distintas ramas del saber, desarrollar actividades de vinculación con la sociedad y las comunidades, y automatizar los procesos de gestión administrativa para favorecer la toma de decisiones. Las TI son parte central de la mejora de las Instituciones de Educación Superior (Lau \& Yuen, 2014) y repercuten tanto en el modo de gestionar como en las funciones sustantivas: docencia, investigación y gestión del conocimiento (Yassin, et al., 2013).

El compromiso de la nueva universidad ecuatoriana con los aspectos tratados anteriormente queda reflejado en la Ley Orgánica de Educación Superior, aprobada en el año 2010, en el título V relativo a la Calidad de la Educación, cuando en el capítulo 1 refiere el principio de calidad como "la búsqueda constante y sistemática de la excelencia, la pertinencia, producción óptima, transmisión del conocimiento (...)" (LOES, 2010, p. 17). En el Art. 94 se define la evaluación de la calidad como: "El proceso para determinar las condiciones de la institución, carrera o programa académico, mediante la recopilación sistemática de datos cuantitativos y cualitativos que permitan emitir un juicio o diagnóstico, analizando sus componentes, funciones, procesos, a fin de que sus resultados sirvan para reformar y mejorar el programa de estudios, carrera o institución. La evaluación de la calidad es un proceso permanente y supone un seguimiento continuo" (Ibídem, p. 17).

A juicio de los autores, la gestión institucional se sustenta en cuatro ejes básicos: i) Academia; ii) Investigación; iii) Vinculación con la sociedad; y iv) Administración

1. Academia: el modelo educativo, didáctico y pedagógico se proyecta hacia el logro de altos estándares de calidad en la gestión de la educación superior. En especial, promueve la investigación científica, la innovación tecnológica y empresarial, en un proceso integral, donde el conocimiento se concibe en construcción y transformación permanente y el estudiante es responsable de su propio aprendizaje.

2. Investigación: La investigación es el eje articulador de todo el proceso de formación de los futuros profesionales

3.

4. Vinculación con la sociedad: El vínculo con la sociedad se produce de forma constante a través de los procesos de investigación, la realización de prácticas pre-profesionales y proyectos de vinculación con la comunidad.

5. Administración: Es esencial para garantizar la sostenibilidad económica de la universidad y apoyar el desarrollo ininterrumpido de sus procesos sustantivos. 
La universidad es una organización que funciona como un sistema, lo que significa que el todo es superior a la suma de las partes. En tal sentido, la información debe ser entendida como una posibilidad de reducir la incertidumbre y de apoyo al proceso de toma de decisiones. Luego, los sistemas de información deben articular una visión sistémica de la universidad para no quedar reducidos a simples bases de datos que no dan cuenta de la verdadera dinámica organizacional. Para planificar acertadamente no basta con elaborar planes bien fundamentados, también es necesario controlar sistemáticamente y medir el grado de cumplimiento de los objetivos estratégicos. Es aquí donde desempeñan un papel muy importante las variables estratégicas, los indicadores y los sistemas de información, como una vía o alternativa para superar los enfoques tradicionalistas y desarrollar una cultura organizacional potenciadora de la gestión universitaria.

El sistema universitario experimenta en los últimos años, un cambio cualitativo y cuantitativo importante en el que cada vez, se hace más evidente una mayor exigencia social y de la comunidad universitaria para mejorar la calidad de las instituciones. La Universidad de Otavalo dedica una atención preferente a la mejora de la calidad de los procesos que lleva a cabo- estratégicos, misionales y de apoyo- a tres niveles distintos: institucional, de las unidades organizativas y de las personas. Los pilares esenciales del sistema de calidad se muestran de forma resumida en la tabla 1. Las prioridades se establecen en el orden siguiente: la planificación institucional, los procesos, la evaluación, y el Sistema de Información Estratégica para la Gestión Universitaria (SIE-GU)

Tabla 1: Sistema de Calidad de la Universidad de Otavalo

\section{Pilares del sistema de calidad de la Universidad de Otavalo}

\begin{tabular}{|c|c|c|}
\hline Planificación & Sistema de información estratégica & Evaluación \\
\hline $\begin{array}{l}\text { Plan Estratégico de Desarrollo } \\
\text { Institucional (PEDI-2015-20) } \\
\text { Áreas de Resultados Clave: } \\
\text { a) Claustro de Profesores } \\
\text { b) Formación Académica } \\
\text { c) Investigación } \\
\text { d) Vinculación con la Sociedad } \\
\text { e) Gestión Universitaria }\end{array}$ & $\begin{array}{l}\text { Identificación de los procesos y } \\
\text { actividades en las unidades } \\
\text { organizativas } \\
\text { a) Estratégicos } \\
\text { b) Claves } \\
\text { c) Apoyo }\end{array}$ & $\begin{array}{l}\text { Modelo de Evaluación Institucional } \\
\text { Criterios: } \\
\text { a) Organización } \\
\text { b) Academia } \\
\text { c) Investigación } \\
\text { d) Vinculación con la Sociedad } \\
\text { e) Recursos e Infraestructura } \\
\text { f) Estudiantes }\end{array}$ \\
\hline $\begin{array}{l}\text { Plan Operativo Anual de la } \\
\text { institución (POA) } \\
\text { Áreas de Resultados Clave: } \\
\text { a) Claustro de Profesores } \\
\text { b) Formación Académica } \\
\text { c) Investigación } \\
\text { d) Vinculación con la Sociedad } \\
\text { e) Gestión Universitaria } \\
\end{array}$ & $\begin{array}{l}\text { Sistema de Información Estratégica } \\
\text { aplicado a la Gestión Universitaria } \\
\text { (SIE-GU) } \\
\text { Módulos: } \\
\text { a) Academia } \\
\text { b) Investigación } \\
\text { c) Vinculación con la Sociedad } \\
\text { d) Administración }\end{array}$ & $\begin{array}{l}\text { Modelo de Evaluación de Carreras } \\
\text { Criterios: } \\
\text { a) Pertinencia } \\
\text { b) Plan curricular } \\
\text { c) Academia } \\
\text { d) Ambiente institucional } \\
\text { e) Estudiantes }\end{array}$ \\
\hline $\begin{array}{l}\text { Planes operativos anuales por } \\
\text { unidades organizativas } \\
\text { a) Académicas } \\
\text { b) Administrativas }\end{array}$ & $\begin{array}{l}\text { Sistema de Monitoreo y Evaluación de } \\
\text { la Planificación Estratégica (SMEPE) } \\
\text { Módulos: } \\
\text { a) Registro } \\
\text { b) Monitoreo } \\
\text { c) Evaluación } \\
\text { d) Ayuda }\end{array}$ & $\begin{array}{l}\text { Evaluación individual del desempeño } \\
\text { a) Docencia } \\
\text { b) Investigación } \\
\text { c) Gestión }\end{array}$ \\
\hline $\begin{array}{l}\text { Plan de Trabajo Individual } \\
\text { a) Docencia } \\
\text { b) Investigación } \\
\text { c) Gestión }\end{array}$ & $\begin{array}{l}\text { Sistema de Información para la } \\
\text { Evaluación Institucional } \\
\text { Módulos: } \\
\text { a) Gestión de la evaluación } \\
\text { b) Información para la evaluación }\end{array}$ & $\begin{array}{l}\text { Planes de Mejora: toma de decisiones } \\
\text { a) Planes de Mejora: institucional, } \\
\text { carrera, individuales. } \\
\text { b) Optimización de los procesos } \\
\text { dentro de la organización }\end{array}$ \\
\hline
\end{tabular}

\section{La planificación institucional}

La Universidad de Otavalo adopta un modelo de gestión universitaria orientado a las estrategias de desarrollo, donde predomina la dirección estratégica. Para ejecutarlo se basa en la propuesta de la Pirámide del Desarrollo Universitario (PEDI, 2015-2020), en la que se integran las áreas de resultados clave, las restricciones de desarrollo, el contexto institucional y la dirección estratégica de la organización. Con la finalidad de formular y ejecutar el Plan Estratégico de Desarrollo Institucional 2015-2020, la universidad implementó una metodología participativa que incluyó distintos niveles de interacción de toda la comunidad universitaria y fueron consideradas las experiencias acumuladas en la ejecución de planes anteriores, así como las recomendaciones de política educativa de organismos nacionales e internacionales. El alineamiento estratégico formó parte de propio modelo (Barra, 2015). Los pasos metodológicos que se 
siguieron en el proceso de planificación estratégica se estructuraron de modo coherente y participativo y abarcaron el área de las informática, pues es conocido ya, que la planeación estratégica de las TI logra generar destacados avances en las áreas en que se implementa (Arvidsson, et al., 2014).

\section{Los procesos}

Sin dudas, contribuyen a articular la planificación con la evaluación y los criterios e indicadores a considerar en los modelos de evaluación. Para desarrollar el sistema de información estratégica es necesario identificar previamente los procesos estratégicos, claves y de apoyo, considerando que la incorporación de tecnología en la ejecución de los procesos de la institución se realiza en base a una planificación corporativa, alineada a los objetivos institucionales y con un enfoque de soporte efectivo en dichos procesos. La etapa de diagramación es fundamental para comprender un proceso, es primaria para el desarrollo del software (Magaña, 2012) y clave para representar de manera estructurada el flujo de trabajo y definir sus participantes (Espinoza y López, 2013)

\section{La evaluación}

Desde hace algún tiempo, la Universidad de Otavalo se enfoca en la gestión de la evaluación de sus actividades, y desarrolla una cultura de la calidad y la rendición de cuentas. La propia dinámica del entorno propicia la evaluación permanente de objetivos y resultados, en lugar de simples constataciones y el paso de la evaluación interna a la externa, considerando la evaluación institucional, de carrera y del desempeño individual (Almuiñas, et al., 2013). Para ello, la UO tiene implantados diversos sistemas de evaluación sistemática por ámbitos de actividades, estrechamente relacionados con la planificación estratégica, la planificación operativa y la gestión de los procesos universitarios.

\section{EI Sistema de Información Estratégica para la Gestión Universitaria (SIE-GU)}

En el contexto universitario, el SIE-GU permite apoyar el normal desarrollo de los procesos universitarios y automatizar las actividades más importantes de la gestión universitaria, para fundamentar la toma de decisiones. Es recomendable que las TI sean utilizadas lo más transversalmente posible al conjunto de procesos que desarrolla la institución, para poder lograr soluciones integradas de amplia base en toda la organización (una única base de datos corporativa, un solo sistema de correo electrónico, un sitio Web institucional, una intranet integrada a un entorno virtual de aprendizaje), sin duplicar esfuerzos y con el mayor grado de eficacia en la utilización de los recursos.

\section{METODOLOGÍA}

Para lograr coherencia entre la propuesta del SIE-GU con los pilares de calidad de la UO, se diseñó una metodología de trabajo que consta de diez etapas: (1) Evaluación de la planificación estratégica institucional: Delimitación del objeto de estudio, la estructura de la organización y el análisis de su ambiente externo e interno (2) Examen de la información disponible en la organización: Políticas y procedimientos, modelo de gestión, manuales de procedimientos específicos, entre otros; (3) Identificación de la información necesaria para la elaboración del SIE-GU; (4) Balance de información: compatibilización de la información disponible y necesaria; (5) Identificación de los procesos de la organización: Manual de procesos, entradas, salidas. Procesos estratégicos, claves y de apoyo; (6) Construcción del equipo de trabajo: Encargado de analizar las principales metodologías de trabajo, resaltando el enfoque participativo, donde el diseño y desarrollo de la entidad están centrados en el usuario (Briede \& Mora, 2013); (7) Definición de los componentes del SIE-GU; (8) Elaboración de los planes estratégicos para la implementación de las aplicaciones informáticas; (9) Evaluación de los resultados: Incluye la validación del sistema y la satisfacción de los usuarios; y (10) Examen de alternativas de mejora del SIE-GU.

La investigación comenzó como un estudio de tipo exploratorio, realizando una minuciosa revisión bibliográfica sobre los sistemas de información y sus aplicaciones en Ecuador y el resto del mundo. Partiendo de la problemática específica de la UO, se definió como variable dependiente la caracterización del SIE-GU en función de: la planificación, la evaluación institucional y la toma de decisiones (variable independiente). El proceso investigativo tuvo sus inicio en el año 2012, con aplicaciones informáticas dispersas, las que se integraron parcialmente en función de necesidades operativas y su propio desarrollo condicionó la necesidad de avanzar a un estadio superior, por lo que en el año 2014 comenzó el desarrollo del SIE, cuya culminación está prevista para el año 2018. Desde el punto de vista ético, la organización se enfoca en la calidad de la recolección de los datos y en divulgar los resultados mediante eventos científicos, publicación de los resultados y participación en redes. No obstaste, el énfasis fundamental está en la propia aplicación por su notable contribución al mejoramiento organizacional, con beneficios para la toma de decisiones operativas y estratégicas en materia de gestión universitaria. 
Considerando que el objeto de estudio de está enmarcado en la gestión de los procesos universitarios y específicamente, el campo de acción vinculado con los sistemas de información estratégica aplicados a la gestión universitaria para apoyar la planificación, evaluación y toma de decisiones en la Universidad de Otavalo, a continuación se describe brevemente la institución. En la sesión del 17 de diciembre del año 2002, el Congreso Nacional de la República del Ecuador dispuso la ley que amparó su fundación. La UO es una institución de educación superior particular, intercultural y autofinanciada. Su instrumento central de gestión es el Plan Estratégico de Desarrollo Institucional, elaborado para el período 2015-20120, en el que están definidas las áreas de resultados claves siguientes: claustro de profesores, formación académica, investigación, vinculación con la sociedad y gestión universitaria. El plan estratégico considera como uno de sus ejes principales el perfeccionamiento del modelo de gestión, para lo cual se apoya en el SIE-GU como uno de sus componentes esenciales. A su vez, se desagrega en los planes operativos anuales hasta llegar al plan de trabajo individual del profesor.

Al finalizar el año 2014, en el organigrama funcional de la institución se definieron veintitrés áreas funcionales. El Consejo Universitario es el máximo organismo ejecutivo colegiado de la universidad y el rectorado ejerce la dirección de dos áreas fundamentales: el Vicerrectorado Académico, al que se subordinan siete carreras: Desarrollo Social y Cultural, Comercio Exterior y Finanzas, Diseño Gráfico, Turismo, Administración de Empresas, Informática y Derecho; y la Dirección General Administrativa, a la que se subordinan la mayoría de las restantes unidades organizativas. El claustro lo forman 38 profesores, de ellos 27 a tiempo completo y 11 a tiempo parcial, de los cuales el $80 \%$ posee título de cuarto nivel y cinco son doctores en ciencias. La matrícula es de 415 estudiantes, por lo que es una institución muy pequeña, pero de mucho significado cultural y socioeconómico para la ciudad de Otavalo y su entorno.

En el proceso investigativo se aplicaron tres cuestionarios. El primero para identificar las características y el contenido que debe tener un SIE pertinente para la UO, dadas sus particularidades y tamaño. EI segundo, para conocer con más detalles la información disponible y necesaria para alcanzar las metas propuestas y el tercero orientado a precisar el sistema de indicadores más apropiado para desarrollar la planificación estratégica y operativa, la evaluación institucional y la toma de decisiones.

Los tres cuestionarios fueron aplicados a los directivos de las 23 áreas académicas y administrativas de la organización, de las cuales siete corresponden a direcciones de carreras, de modo que se abarcó el total de la población requerida para los fines propuestos. Los cuestionarios fueron aplicados a finales del año 2014 , en el momento en que las aplicaciones del SGAA alcanzaron un grado de madurez determinado y se decide evolucionar a un estadio superior: el SIE-GU. Debido a que el sistema no está implementado en su totalidad aún están pendientes de realizar cuestionarios relativos a su percepción por parte de los usuarios, incluyendo a estudiantes. Además, se realizó un minucioso análisis de la documentación jurídica de la institución, la planificación estratégica, los modelos de evaluación y otros documentos. Los encuestados valoraron aspectos relacionados con la información que podría ser útil manejar mediante el uso de las TI. La compatibilización permitió identificar la información estratégica de la institución en los ámbitos de la academia, la investigación, la vinculación con la sociedad y la gestión administrativa.

El siguiente paso en el análisis consistió en construir el mapa de procesos de la universidad. Fueron definidos los procesos estratégicos: gestión institucional, gestión de las comunicaciones e informatización, gestión del talento humano, entre otros; los procesos claves: formación, investigación y vinculación con la sociedad; y los procesos de apoyo: gestión administrativa-financiera, gestión del bienestar universitario, gestión de los sistemas de información, y otros. Para cada uno de ellos se definieron las actividades a realizar y finalmente se solicitaron los diagramas de flujo a cada una de las áreas académicas y administrativas.

Al tener definidas las necesidades, áreas principales objeto de estudio, procesos y actividades, se conformó un equipo de trabajo con los conocimientos necesarios sobre TI y su importancia para el mejoramiento de los estándares de calidad en la organización. El mismo está conformado por un director del proyecto (Director General Administrativo), dos gestores de proyectos, uno de los cuales es el Director de Planificación y cuatro analistas de sistemas, incluyendo a su Director. El equipo definió el estado en el que se encontraba la universidad y sus necesidades de información haciendo uso de métodos para la recolección y análisis de datos y en los últimos cinco años laboró en el análisis de la problemática de la modernización de las tecnologías de la información aplicadas a los procesos de gestión universitaria para mejorar el desempeño institucional. Además, en estrecha colaboración con los directivos definió las características y el contenido del SIE-GU, para sustentar de manera efectiva la planificación, evaluación y toma de decisiones.

Para evaluar el nivel de implementación del SIE-GU, se realizó un cuestionario en junio de 2016, donde se solicitó a cada uno de los directivos su valoración sobre el nivel de utilización e implementación de las aplicaciones informáticas pertenecientes a cada uno de los cuatro por módulos del sistema, otorgando una 
calificación de 1 a 5 (ver Tabla 2). Para ello se utilizó una escala común, con los siguientes niveles: 1 = No se está utilizando esta aplicación de TI en la universidad; 2 = Está prevista la utilización de la aplicación de TI, pero no se ha implantado todavía; 3 = Esta aplicación de TI está en proceso de implantación; 4 = Esta aplicación de TI está implantada y requiere de ser perfeccionada; y 5 = Esta aplicación de TI está en pleno funcionamiento y continúa su actualización.

\section{RESULTADOS}

EI SIE-GU (ver Figura 1), está formado por cuatro módulos principales con sus respectivas aplicaciones, responde al modelo cliente-servidor y considera los procesos más importantes de la institución y sus respectivas actividades; con acceso pleno a intranet e internet; es único e integrado y favorece la participación de los actores implicados, aportando información valiosa orientada a la toma de decisiones para el mejoramiento continuo de la calidad y los resultados de la gestión universitaria. EI SIE-GU abarca un espacio digital, a través de las plataformas unificadoras (Zhan, et al., 2003). Son muy importantes las características, de acuerdo a los objetivos propuestos ( $\mathrm{Ng}$, et al., 2011). En el caso del SIE-GU, sus características pueden resumirse en: (1) Módulos personalizados; (2) Información centralizada; (3) Administración de ususarios; (4) Administración de roles y permisos; (5) Reportes on-line; (6) Estructura cliente-servidor; (7) Seguimiento continuo; y (8) Estándares de calidad de sofware.

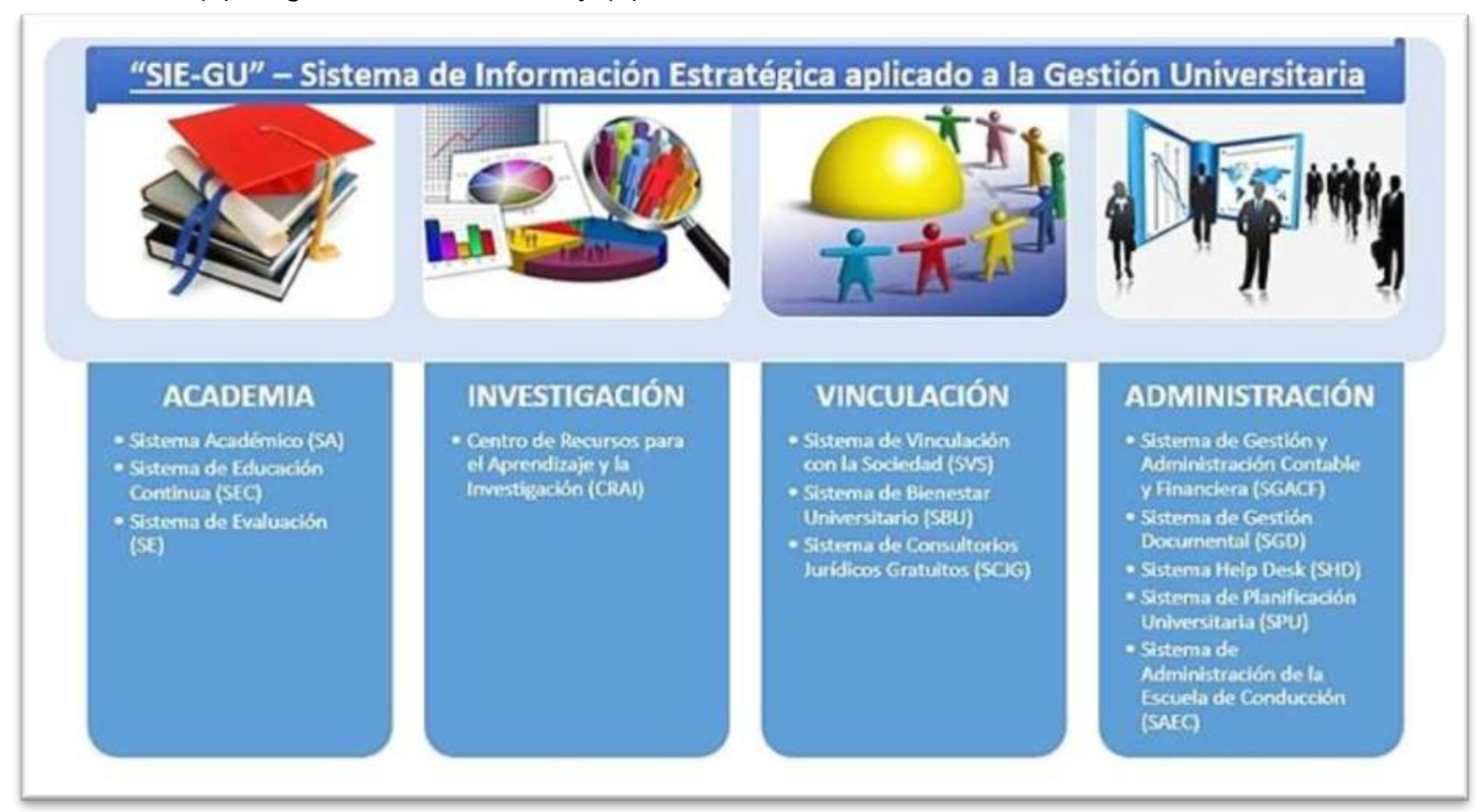

Fig. 1: Módulos del SIE-GU

El SIE-GU, al ofrecer información sobre el Plan Estratégico de Desarrollo Institucional (ver Figura 2), incluye: la misión, la visión, los valores compartidos, la metodología de elaboración y las herramientas aplicadas, las áreas de resultado clave, objetivos estratégicos, estrategias y planes de acción, la matriz de objetivos estratégicos, los programas, proyectos y actividades, el sistema de indicadores utilizado para medir la gestión universitaria, el presupuesto y el plan de monitoreo y evaluación. Como parte del proceso de evaluación del cumplimiento de los objetivos, el SIE-GU apoya el proceso del chequeo del cumplimiento parcial y final de los resultados para cada uno de los ejes estratégicos, programas y proyectos. Para controlar el nivel de cumplimiento de los indicadores se apoya en un sistema de alerta basado en una gama de colores, expresado en un cuadro de mando integral, lo que representa una gran ayuda para los directivos en el análisis de los resultados y la toma de decisiones relacionadas con el Plan Operativo Anual y el modelo de evaluación.

La aplicación referida a la evaluación institucional se presenta en dos secciones: información y gestión. En la primera sección se carga al sistema la información por criterios, subcriterios e indicadores establecidos en el modelo aplicado por el Consejo de Evaluación, Acreditación y Aseguramiento de la Calidad de la Educación Superior (CEAACES) y en cada indicador se reportan las evidencias que lo sustentan con su porciento de cumplimiento. Con respecto a la gestión el sistema facilita evaluar con agilidad los niveles porcentuales de cumplimiento de cada uno de los indicadores, subcriterios y criterios con respecto al patrón de calidad, lo que es de mucha utilidad para los directivos en los seguimientos diarios, semanales, mensuales y anuales y para la obtención de información útil para la toma de decisiones estratégicas y operativas. 
Mediante el SIE-GU se realiza la evaluación del desempeño de los docentes a tiempo completo y parcial. Los propios profesores realizan su autoevaluación con ayuda del sistema, los estudiantes efectúan la heteroevaluación de sus profesores, los pares evalúan a los docentes y los directivos valoran el cumplimiento de los planes de trabajo individuales de sus subordinados. Finalmente, a través del sistema se realiza la evaluación integral considerando las funciones reglamentadas: docencia, investigación y gestión.

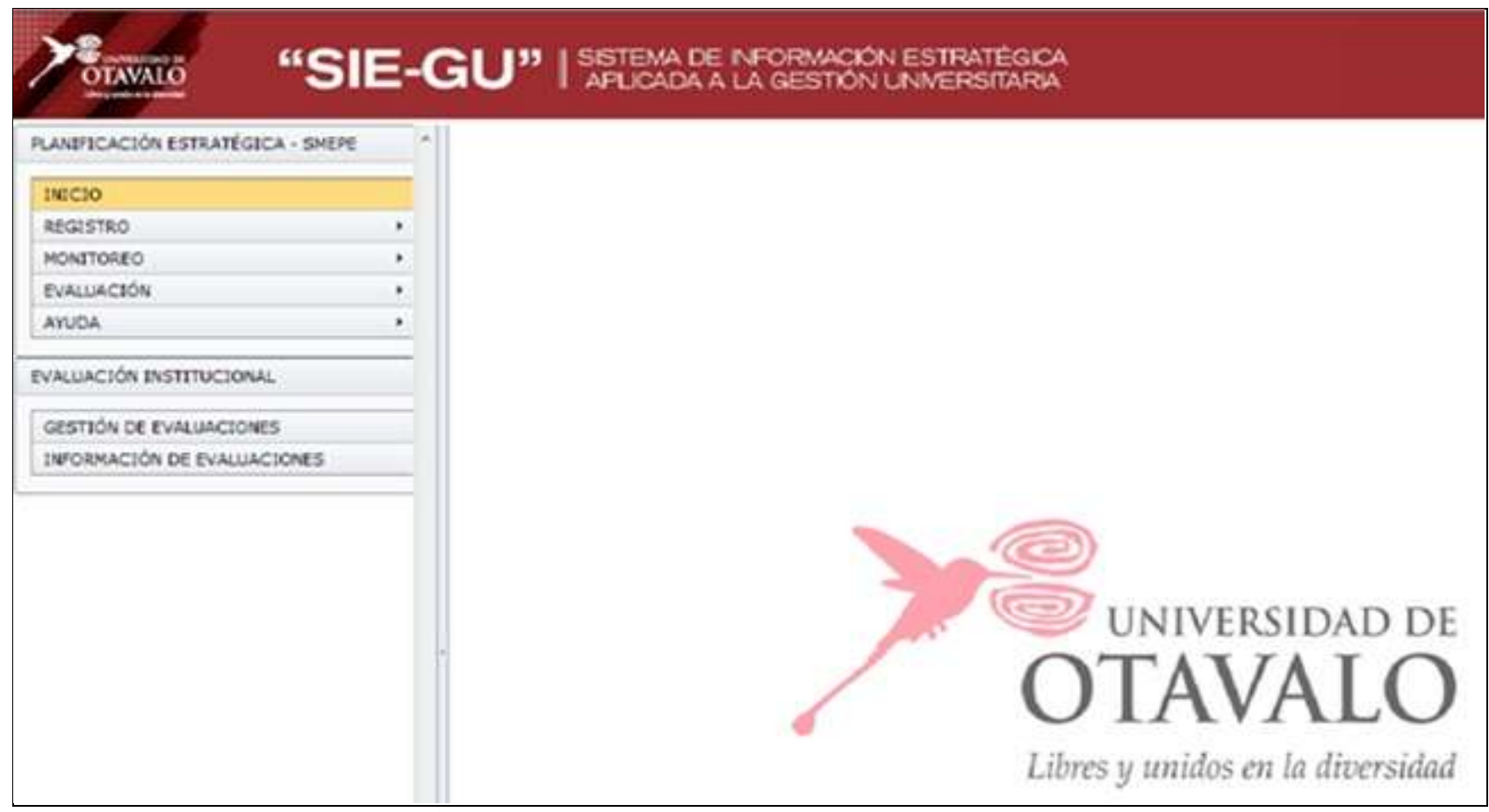

Fig. 2: SIE-GU: Planificación Estratégica (SMPE) y Evaluación Institucional

Tabla 2: Módulos del SIE-GU y sus aplicaciones

\begin{tabular}{|c|c|c|c|c|c|c|}
\hline$N^{\circ}$ & Módulos del SIE-GU y aplicaciones & 1 & 2 & 3 & 4 & 5 \\
\hline \multicolumn{7}{|c|}{ Academia } \\
\hline 1 & Sistema Académico- SA & & & & & \\
\hline 2 & Sistema de Actualización de Datos- SADTS & & & & & \\
\hline 3 & Ficha Socioeconómica- FSE & & & & & \\
\hline 4 & Repositorio Digital- DSPACE & & & & & \\
\hline 5 & Sistema de Seguimiento del Sílabo-SSS & & & & & \\
\hline 6 & Sistema de Homologaciones-SH & & & & & \\
\hline 7 & Sistema de Evaluación a Docentes- SED & & & & & \\
\hline \multicolumn{7}{|c|}{ Investigación } \\
\hline 8 & Entorno Virtual de Aprendizaje- EVA & & & & & \\
\hline 9 & Sistema de Administración de Bibliotecas- SAB & & & & & \\
\hline 10 & Sistema de Proyectos-SP & & & & & \\
\hline \multicolumn{7}{|c|}{ Vinculación } \\
\hline 11 & Sistema de Administración de Herramientas Psicológicas- SAHP & & & & & \\
\hline 12 & Sistema de Encuestas- SE & & & & & \\
\hline 13 & Sistema de Graduados- SG & & & & & \\
\hline 14 & Sistema Consultorios Jurídicos Gratuitos- SCJG & & & & & \\
\hline \multicolumn{7}{|c|}{ Administración } \\
\hline 15 & Sistema de Registro de Personal- SRP & & & & & \\
\hline 16 & Sistema de Archivos e Inventarios- SAI & & & & & \\
\hline 17 & Sistema Contable-SC & & & & & \\
\hline 18 & Sistema de Gestión Documental-Help Desk (SHD) & & & & & \\
\hline 19 & Sistema de Planificación Universitaria (SPU) & & & & & \\
\hline 20 & Sistema de Evaluación Interna- SEI & & & & & \\
\hline 21 & Sistema de Escuela de Conducción (SEC) & & & & & \\
\hline
\end{tabular}


Al analizar y evaluar el grado de implementación de las aplicaciones (ver Tabla 2), se puede apreciar que la mayoría está en pleno funcionamiento y continúa su actualización o están implantadas y requieren ser perfeccionadas. Finalmente, se elaboró un plan de alternativas de mejora donde destaca el plan estratégico para la culminación de las aplicaciones no concluidas y la necesidad de continuar perfeccionando su integración, así como la eliminación de las posibles redundancias.

Los principales aportes que resaltan de la elaboración del SIE-GU son los siguientes: (i) Una contribución teórica, enmarcada en la fundamentación del diseño e implementación de un SIE-GU, de carácter integrado, considerando los procesos más importantes de la institución y sus interrelaciones; (ii) Una contribución metodológica, al proponer una metodología fundamentada científicamente para la elaboración de un SIEGU, incluyendo etapas, contenido, pasos necesarios y estructura requerida para aplicarlo a la realidad concreta y específica de la UO; (iii) Una contribución práctica mediante la implementación del SIE-GU, el cual se apoya en un programa informático de consulta permanente por los actores involucrados, y que incluye a toda la comunidad universitaria.

\section{DISCUSIÓN}

El SIE-GU tiene sus antecedentes en un Sistema de Gestión Académica y Administrativa (SGAA) implementado en los últimos años en la Universidad de Otavalo. Independientemente de los elementos positivos que resaltan de las aplicaciones informáticas y del reconocimiento social por esta experiencia, el proyecto SGAA dejó algunos vacíos importantes. De la valoración crítica realizada al SGAA, surgió la necesidad de avanzar a un estadio superior, mediante el diseño e implementación del SIE-GU, cuyas características y contenido favorecen de manera efectiva la toma de decisiones estratégicas, tácticas y operativas en materia de planificación estratégica y operativa, autoevaluación institucional, evaluación del desempeño y otras dimensiones de la gestión universitaria.

El principal vacío identificado en las aplicaciones del SGAA estuvo dado por la no existencia de una plataforma centralizada de información en línea para la gestión. Por ello, existen sólidos argumentos que refuerzan la necesidad de fundamentar teórica y metodológicamente la necesidad de implementar un SIEGU. Las continuas amenazas a que está sometida la UO ante los retos actuales y futuros de la educación superior ecuatoriana imponen la necesidad y urgencia de consolidar una cultura de gestión de la calidad y a ello contribuye la utilización de un sistema integrado para apoyar el desarrollo de la planificación, la ejecución de los procesos universitarios, la evaluación institucional y su adecuada interrelación con las exigencias del patrón de calidad definido por el organismo externo evaluador.

En este sentido, la evidencia empírica permite ratificar que en la mayoría de las universidades existen pocas experiencias en el diseño e implementación de un SIE-GU. Cuando existen, generalmente se trata de aplicaciones incompletas debido a que no integran los cuatro ejes básicos mencionados. Haber logrado un nivel de implementación e interrelación de las aplicaciones informáticas concluidas es el resultado más importante de la investigación realizada y se convierte en un desafío permanente para la asimilación de otras experiencias nacionales e internacionales, la integración plena de las aplicaciones y el monitoreo permanente del grado de satisfacción de los clientes internos y externos, considerando los grandes volúmenes de información que se procesan sistemáticamente en una universidad.

\section{CONCLUSIONES}

La implementación práctica de las aplicaciones informáticas concluidas y que forman parte del contenido del SIE-GU, favorecen la automatización de la mayoría de los procesos y actividades de la institución. El plan veintiuna aplicaciones pertenecientes a cuatro módulos, de las cuales catorce están en pleno funcionamiento, cuatro están implantadas pero requieren ser perfeccionadas y tres están en proceso de implantación. Todas las aplicaciones ofrecen reportes de salida que nutren los indicadores de la planificación y evaluación institucional, el cuadro de mando integral y la evaluación del desempeño de los docentes a tiempo completo y parcial, de modo que contribuyen a la toma de decisiones estratégicas, operativas y tácticas en esas áreas de la gestión universitaria. El sistema está en un 91,4 \% de implementación y contribuye a agilizar la obtención de información útil para realizar acciones correctivas orientadas al mejoramiento continuo de la calidad de la gestión universitaria, lo cual es muy importante para una universidad como la de Otavalo, con un alto grado de alfabetización digital.

\section{REFERENCIAS}

Ali, R. H., Strategic is Planning Practices: A Comparative Study of Malaysia and New Zealand. ProcediaSocial and Behavioral Sciences, 164, 516-521 (2014)

Almuiñas, J.L. y Galarza J. (Eds). La evaluación del desempeño del docente universitario: experiencias institucionales y nacionales. Guayaquil, Ecuador, Senefelder, https://goo.gl/Nz6TnU (2013) 
Arvidsson, V. H., Information systems use as strategy practice: A multi-dimensional view of strategic information system implementation and use, The Journal of Strategic Information Systems, 23(1), 45-61 (2014)

Barra, A. M., Alineamiento Estratégico Sectorial: Caso de Estudio Aplicado a una Universidad Chilena, Formación Universitaria, 8(3), 3-12 (2015)

Briede, J. C., Mora, M. L., Propuesta evaluativa para el Taller de Diseño Centrado en el Usuario (DCU), en la Carrera de Diseño Industrial de la Universidad del Bío-Bío, Chile, Formación Universitaria 6(2), 33-42 (2013)

Dastan, D.C, The Effects of Information Technology Supported Education on Strategic Decision Making: An Empirical Study, Procedia-Social and Behavioral Sciences, 24, 1134-1142 (2011)

Espinosa, Y., López, C. R., Business Process Modeling: Evolution of the Concept in a University Context, Computación y Sistemas, 17 (1), 79-83 (2013)

Hosseini, M. H., Karimzadegan, D., y Sazvar, A., Identification of Management Information System (MIS) strategies barriers in higher education institutions through multi-criteria decision making (MCDM) approach: Case study of Ferdowsi University of Mashhad, Educational Research and Reviews, 7(5), 111-120 (2012)

Lau, W. W., y Yuen, A. H., Developing and validating of a perceived ICT literacy scale for junior secondary school students: Pedagogical and educational contributions, Computers \& Education, 78, 1-9 (2014)

LOES. Ley Orgánica de la Educación Superior. Presidencia de la República del Ecuador. Documento digitalizado de la publicación original. Lexis S.A. (2010)

Magaña-Echeverría, M. A., Santana-Mancilla, P. C., y De la Rocha-Cazares, V. M., An Educational Management Information System to Support Institutional Planning at the University of Colima, ProcediaSocial and Behavioral Sciences, 55, 1168-1174 (2012)

Mansfield, R., "Changes in Information Technology, Organizational Design and Managerial Control", en Piercy, N., The Management Implications of New Information Technology, London, Croom Helm (1984)

Martelo, R. J., Ponce, A. L., y Acuña, F., Guía Metodológica para el Diseño de un Plan Estratégico Informático en Instituciones de Educación Superior, Formación Universitaria, 9(1), 91-98 (2016)

Martínez, R., Estudio y diseño de un sistema de información gerencial universitario integrado (SIGUI), (2006)

Martín-Gutiérrez J., Fabiani P., Benesova W., Meneses M.D., Mora C., Augmented reality to promote collaborative and autonomous learning in higher education, Computers in Human Behavior, 51, 752-761 (2015)

Ng, S. C., Wong, C. K., Lee, T. S., y Lee, F. Y., Design of an Agent-based Academic Information System for Effective Education Management, Information Technology Journal, 10(9), 1784-1788 (2011)

PEDI 2015-2020. Plan Estratégico de Desarrollo Institucional 2015-2020, Universidad de Otavalo, Ecuador. (En línea: https://goo.gl/2vIVPJ, acceso: 17 junio 2016), Otavalo (2016)

Puello, P., Cabarcas, A., y Martelo, R., Sistema de información gerencial para la administración de recursos educativos, Formación Universitaria, 6(5), 13-20 (2013)

Senn, J.A., Análisis y Diseño de Sistemas de Información, México, McGraw-Hill (1992)

UNESCO. Conferencia Mundial sobre la Educación Superior. La educación superior en el siglo XXI. Visión y Acción. Informe Final. Tomo I. UNESCO, París, Francia, https://goo.gl/QFLHjp (1998)

Whitten, J., Bentley. L.D., y K.C. Dittman, Sistem análisis \& design methods citado por Fernández Vicenç, 2006: Desarrollo de sistemas de información: una metodología basada en el modelado (2004)

Yassin, F. S., The Influence of Organizational Factors on Knowledge Sharing Using ICT among Teachers, Procedia Technology, 11, 272-280 (2013)

Zhan, H. F., Lee, W. B., Cheung, C. F., Kwok, S. K., y Gu, X. J., A web-based collaborative product design platform for dispersed network manufacturing, Journal of Materials Processing Technology, 138(1-3), 600$604(2003)$ 